\title{
1 Glucocorticoids mediate egg rejection in a brood parasite host
}

3 Mikus Abolins-Abols* and Mark E. Hauber

5 Department of Evolution, Ecology, and Behavior, School of Integrative Biology, University of Illinois,

6 Urbana-Champaign, IL 61801, USA

7

$8 \quad *$ Corresponding author email: abolins@illinois.edu

\section{Abstract}

11 Avian brood parasites and their hosts are engaged in a coevolutionary battle that can result in the

12 evolution of sophisticated trickery by parasites and novel defence behaviours in hosts. Despite the clear

13 evolutionary and ecological significance of host behaviour, however, we know very little about the

14 mechanisms that regulate host defences, which limits our understanding of both inter- and intraspecific

15 variation in host responses to parasitism. Here we tested whether corticosterone, a hormone known to be

16 upregulated in hosts exposed to parasitism, also mediates one of the most frequent host defences - the

17 rejection of foreign eggs. We experimentally reduced corticosterone levels in free-living brood parasite

18 hosts, American robins Turdus migratorius, using mitotane and found that the likelihood of model egg

19 rejection was significantly lower in the mitotane-treated birds relative to the sham-treated birds. These

20 results demonstrate a causal link between glucocorticoids and egg rejection in hosts of avian brood

21 parasites, but the physiological and sensory-cognitive pathways that regulate this effect remain unknown.

23 Keywords: avian brood parasitism, egg rejection, corticosterone

\section{1. Introduction}


26 In obligate avian brood parasitism, parasites exploit host parental care by forcing the foster parents to

27 provision unrelated offspring [1,2]. To mitigate the negative fitness consequences of parasitism, hosts can

28 evolve resistance strategies, such as the ability to recognize and reject foreign eggs or young in the nest

$29[3,4]$, or tolerance strategies, such as the ability to withstand the physiological costs of caring for, or

30 coexisting with, a parasitic nestling [5]. Although much is known about the perceptual cues and

31 behavioural responses that hosts employ to recognize and reject brood parasitic stimuli [6], the

32 physiological mechanisms that underlie host responses to brood parasites remain poorly understood [7].

33 A handful of recent studies suggest that host responses to avian brood parasitism are, in part,

34 mediated by steroid hormones [8]. For example, host parents caring for a parasitic chick show higher

35 stress-induced corticosterone levels compared to parents caring for only their own young [9]. An increase

36 in the baseline corticosterone levels in hosts can occur directly in response to encountering a foreign egg

37 in the nest [10]. Furthermore, parasitism can alter the endocrine milieu of host offspring, either through an

38 increase in maternally-deposited yolk androgen concentrations [11], or by an increase in the baseline

39 corticosterone levels in host nestlings during competition with parasitic chicks [12]. The physiological

40 and behavioural consequences, and the adaptive value of these hormonal changes, if any, are as of yet

41 unclear, because glucocorticoids in general, and corticosterone in particular, can regulate diverse and even

42 opposing functions [13].

43 Elevated corticosterone levels in hosts in response to foreign eggs suggests a possible role of this

44 hormone in mediating host resistance or tolerance strategies. Here we tested the hypothesis that egg

45 rejection, a widespread host defence against brood parasites, is mediated by circulating corticosterone.

46 Specifically, we experimentally lowered plasma glucocorticoid levels using mitotane injections in an egg-

47 rejecter host species and asked whether this treatment shifted the probability of non-mimetic foreign egg

48 rejection compared to a sham-treatment. Because corticosterone can mediate vigilance in vertebrates [14]

49 and suppress parental behaviours in birds $[15,16]$, we predicted that inhibition of glucocorticoid synthesis

50 would reduce foreign egg rejection. 


\section{Methods}

53 (a) Field site and species

54 We studied wild American robins Turdus migratorius, an occasional host to the obligate brood-parasitic 55 brown-headed cowbirds Molothrus ater, at Wandell's Tree Nurserty in Champaign County, IL, USA, 56 during the summer of 2019 (details of the study area are given in $[17,18]$ ). We searched for robin nests

57 daily. Prior to the experiment, we monitored the content of focal nests every third day, assuming the 58 clutch was complete when the egg number did not change within 24 hrs [19]. For this study, we focused

59 only on female robins, because in this and other species with female-only incubation they are the only sex

60 responsible for egg rejection [20].

61

(b) Treatment validation

63 Mitotane is a glucocorticoid synthesis inhibitor that has been shown to consistently reduce baseline

$64[21,22]$ and stress-induced [22-24] corticosterone levels in birds, often to non-detectable levels. We first

65 tested if the effect of mitotane on corticosterone levels in American robins parallels that seen in other

66 species. We caught wild egg-laying or incubating robin $(n=8)$ females and took a baseline blood sample

67 from the brachial vein within 3 min of capture (mean start time $=130 \mathrm{sec}$; mean end time $=167 \mathrm{sec}$ ).

68 Blood was stored on ice and centrifuged at 8000 RPM within 2 hrs to separate plasma. Plasma samples

69 were kept on ice until frozen at $-80{ }^{\circ} \mathrm{C} 4 \mathrm{hrs}$ later. We then injected the pectoral muscle of 4 females with

$7034 \mathrm{mg}$ mitotane (Sigma-Aldrich, Cat. No. 25925), dissolved in $400 \mu 1$ sterile peanut oil (Acros Organics,

71 Cat. No. 416855000 , dosage $400 \mathrm{mg} / \mathrm{kg}$ ), following guidelines for the maximum mitotane dosage used in

72 other species $[21,22]$. Four sham females were injected with a pure peanut oil vehicle $(400 \mu 1)$.

73 Recapturing wild robins within a day in most cases is not possible, because they become

74 extremely vary of mist nets and humans. We therefore temporarily transferred the injected birds to

75 captivity. We housed birds singly overnight in $40 \times 40 \times 34 \mathrm{~cm}$ cages, providing them with ad libitum

76 water, earthworms, bananas, and crushed dry dog food. The following day, we again collected their blood

77 within 3 min of capture (mean start time $=94 \mathrm{sec}$; mean end time $=120 \mathrm{sec}$ ). Captivity was an intense 
stressor: despite a constant access to food, all birds lost mass, although, on average, they lost less mass in the mitotane $(8.1 \%)$ than in the sham $(14.3 \%)$ treatment (two-tailed $\mathrm{t}$-test, $\mathrm{n}=6, \mathrm{p}=0.01)$. using an enzyme immunoassay (Cayman Chemical, Cat. No. 501320). Validation details and methods for this assay using robin plasma are published elsewhere [25]. All samples were assayed in duplicate on the same plate, with an intraplate coefficient of variation of $6.7 \%$.

(c) Hormone manipulation in the wild

86 We captured incubating robin females $(n=61)$ at their nests using a mist net between 6-10 am, soon after

87 they had completed their clutches (median 2 days, range 0-5 days after clutch completion). We first took a

$88450 \mu \mathrm{l}$ blood sample as part of a different investigation. We then injected the bird either with mitotane or sham treatment, as described above. We also took standard morphometric measurements, including age (using wing feather coloration [26]), mass (nearest g), tarsus (nearest $0.1 \mathrm{~mm}$ ), wing (nearest $\mathrm{mm}$ ), and

91 pectoral muscle condition [27]. We then fitted females with a USGS band and 3 plastic colour bands

92 (Avinet) to facilitate individual identification in the field, following which the birds were released. An

93 unanticipatedly large number of birds abandoned their nests after the injections (see Results and the

94 Ethics statement), which resulted in a substantial reduction in the final sample size ( $\mathrm{n}=37)$ compared to

95 the initial captures.

97 (d) Experimental parasitism with model eggs

98 American robins reject the majority of natural cowbird [28] or model cowbird-like eggs [3], whereas they

99 show more variable responses to egg colours near their rejection threshold, which lies between brown-

100 beige and robin-blue colours [29]. Importantly, robins show variable but individually repeatable responses

101 to deep-blue cowbird-sized model eggs (figure 2 inset; for details, see [30]). We therefore used deep-blue

102 3D-printed eggs to investigate the effect of the injection treatment on egg rejection. 
A day after the mitotane or sham injections, we added one deep-blue model egg to the focal nest.

105 We verified the identity of the female using band colours, and checked the temperature of eggs to

106 establish that the nest was active. We did not remove any robin eggs, because Turdus thrushes show the

107 same response to model eggs regardless of whether their own eggs are removed [31]. We returned to the

108 nest one day after the addition of the model egg to record whether it was accepted (present in the nest) or

109 rejected (missing). We again checked if the nest was active. If the female was not observed during these

110 visits, we returned to the nest later to confirm female identity. Robins do not abandon their nests

111 following experimental parasitism (relative to control eggs; [32]). Depredated nests were excluded from

112 the analysis.

(e) Statistical analyses

115 In the treatment validation study, baseline corticosterone levels were not normally-distributed, therefore 116 we used Mann-Whitney U-tests to assess the differences in hormone levels between mitotane and sham 117 treatments.

118 We found that life history, seasonal, and morphological variables were not different between the 119 treatment groups (all $\mathrm{p}>0.05$, data not shown). Because the treatments were randomized across

120 individuals and time, we tested the effect of the injections on bird behaviour using Fisher's exact tests.

\section{3. Results}

123 In the treatment validation study, females had similar corticosterone levels prior to the injections (two-

124 tailed Mann-Whitney $\mathrm{U}$ test, $\mathrm{n}=8, \mathrm{U}=6, \mathrm{p}=0.686$ ). After a day in captivity, as predicted, the mitotane-

125 treated birds $(n=4)$ showed a smaller increase in corticosterone compared to the sham-treated birds $(n=4$;

126 one-tailed Mann-Whitney $\mathrm{U}$ test, $\mathrm{U}=2, \mathrm{p}=0.057$, figure 1).

127 Twenty-four out of 61 females (39\%) abandoned their nests within a day of the experimental

128 injections. The probability of nest abandonment did not depend on the treatments (two-tailed Fisher's

129 Exact test, $\mathrm{p}=0.601$ ). 
The mitotane-treated birds were significantly more likely to accept the model egg (15 out of 20,

$13175 \%$ ) compared to the sham-treated individuals ( 7 out of 17, 41.2\%; two-tailed Fisher's Exact test, $n=37$,

$132 \mathrm{p}=0.050$, figure 2).

133

\section{4. Discussion}

135 Host adults show elevated baseline and stress-induced corticosterone levels in response to brood

136 parasitism $[9,10]$. Until now, the consequences of this endocrine response on host antiparasitic behaviours

137 have been unknown [8]. Here we experimentally investigated if corticosterone plays a role in mediating

138 host defences against brood parasites. We showed that treatment with mitotane, which inhibits

139 glucocorticoid synthesis, increases the probability of acceptance of a non-mimetic deep-blue model egg

140 by American robins, compared to a sham treatment. This is the first study to establish an endocrine basis

141 of a widespread and effective antiparasitic host defence [7].

142 Hormonal mediation of host resistance against brood parasites has important implications for the

143 ecology and evolution of these behaviours. Specifically, because of the pleiotropic effects of

144 glucocorticoids on host phenotype [33,34; but see 35], and the sensitivity of these hormones to various

145 environmental and social stimuli [36,37], an endocrine basis of host behaviour necessitates that we

146 consider these defences as part of an integrated phenotype. For example, stable individual differences in

147 glucocorticoid levels or the expression of glucocorticoid receptors, or changes in glucocorticoids in

148 response to physical or social challenges, may explain variation in the propensity (or ability) of hosts to

149 respond to brood parasitism. So far, the only study that has investigated such links has found a weak

150 positive association between foreign egg acceptance and baseline corticosterone levels [25]. It is therefore

151 unclear if the increased acceptance of model eggs in response to mitotane in this study is mediated by

152 changes in baseline (as in [10]) or parasitism-related stress-induced [9] corticosterone levels. Indeed, our

153 treatment validation design only allowed us to test if mitotane could alleviate a major increase in

154 corticosterone levels in response to captivity stress. This topic therefore merits future attention. 
The mechanisms through which glucocorticoids affect the probability of egg rejection are unknown. For example, a rise in glucocorticoid levels in response to a parasitic egg (or an adult brood

157 parasite) may activate an action pattern of discrimination, recognition, and rejection [6]. Another

158 possibility is that the effect of glucocorticoids on egg rejection is mediated by a general suppression of

159 affiliative maternal behaviour [8]. Corticosterone has been shown to have a suppressive effect on the

160 parental behaviour across different bird species [16,38; but see 39]. A testable hypothesis is that, in brood

161 parasite hosts, glucocorticoids may suppress affiliative maternal behaviour towards egg-like stimuli,

162 leading to a lower stimulus threshold for foreign-egg rejection. In contrast to this prediction, we found

163 that nest abandonment did not depend on our treatments; however, we consider the nest abandonment to

164 be a response to capture and injection per se, and not to the treatment type. Future studies should consider

165 using a less capture-sensitive host species or less invasive and symmetrical manipulations (both increased

166 vs. suppressed) of hormone levels in wild birds [40,41].

Finally, our study suggests a possible evolutionary scenario for the evolution of host defences.

168 Hosts may first evolve endocrine-regulated tolerance or avoidance mechanisms $[9,10]$, either in response

169 to direct or indirect cues and costs of brood parasitism. These mechanisms may then be co-opted to

170 regulate host defences, such as egg rejection.

171 In summary, we show that a widespread host defence in response to brood parasitism is mediated

172 by glucocorticoid hormones. Future experiments should focus on the physiological and cognitive

173 pathways that regulate this effect, e.g., testing if corticosterone mediates egg rejection through

174 suppressing maternal behaviour in general or by activating specific cognitive action patterns.

175 Furthermore, our research puts host defences in an integrative organismal context, therefore they should

176 be considered in concert with the internal milieu and the stress-ecology of the host species. Finally, a

177 natural next step is to investigate the mechanisms that underlie variation in rejection of natural parasitic

178 eggs in hosts of egg-mimetic brood parasites, to understand how the control of host behaviour evolves in

179 response to increasing parasitic trickery. 
181 Ethics. This study was approved by the animal ethics committee (IACUC) of the University of Illinois

182 (\#17259), and by permits from USA federal (MB08861A-3) and Illinois State agencies (NH19.6279).

183 Despite our best efforts to minimize disturbance at nests, the capture and injection treatments resulted in

184 an unanticipatedly high rate of nest abandonment (39\%). This was much higher than the abandonment

185 due to capture (but not injection) we observed in a previous season (25\%) [25]. To limit negative

186 consequences to the population, we therefore narrowed our study to a single treatment vs. the sham

187 manipulation. Importantly, injected birds were often observed re-nesting, indicating that the effect of

188 injections was not permanent.

190 Data accessibility. The data included in these analyses will be deposited into Dryad upon acceptance.

192 Authors' contributions. MA-A and MEH designed the study, MA-A conducted field and lab work and 193 analysed the data. MA-A and MEH co-wrote the manuscript.

195 Competing interests. We declare that we have no competing interests.

197 Funding. Harley Jones Van Cleave Professorship of the University of Illinois.

199 Acknowledgements. We thank the landowners for permission to work on their properties and C. Goethe 200 for assistance with field work. 


\section{References}

1. Davies NB. 2000 Cuckoos, Cowbirds, and Other Cheats. London: Poyser.

2. Soler M, editor. 2017 Avian brood parasitism: Behaviour, ecology, evolution and coevolution. Cham, Switzerland: Springer.

3. Rothstein SI. 1982 Mechanisms of avian egg recognition: which egg parameters elicit responses by rejecter species? Behav. Ecol. Sociobiol. 11, 229-239. (doi:10.1007/BF00299299)

4. Grim T. 2007 Experimental evidence for chick discrimination without recognition in a brood parasite host. Proc. Biol. Sci. 274, 373-381. (doi:10.1098/rspb.2006.3731)

5. Medina I, Langmore NE. 2016 The evolution of acceptance and tolerance in hosts of avian brood parasites. Biol. Rev. 91, 569-577. (doi:10.1111/brv.12181)

6. Manna T, M. Moskát, Hauber ME. 2017 Cognitive decision rules for egg rejection. In Avian brood parasitism: Behaviour, ecology, evolution and coevolution (ed M Soler), pp. 437-448. Cham, Switzerland: Springer.

7. Avilés JM. 2018 Can hosts tolerate avian brood parasites? An appraisal of mechanisms. Behav. Ecol. 29, 509-519. (doi:10.1093/beheco/arx 150)

8. Abolins-Abols M, Hauber ME. 2018 Host defences against avian brood parasitism: an endocrine perspective. Proc. R. Soc. B Biol. Sci. 285, 20180980. (doi:10.1098/rspb.2018.0980)

9. Mark MM, Rubenstein DR. 2013 Physiological costs and carry-over effects of avian interspecific brood parasitism influence reproductive tradeoffs. Horm. Behav. 63, 717-722. (doi:10.1016/j.yhbeh.2013.03.008)

10. Ruiz-Raya F, Soler M, Abaurrea T, Chastel O, Roncalli G, Ibáñez-Álamo JD. 2018 Hormonal responses to non-mimetic eggs: is brood parasitism a physiological stressor during incubation? Behav. Ecol. Sociobiol. 72, 153. (doi:10.1007/s00265-018-2565-8)

11. Hahn DC, Wingfield JC, Fox DM, Walker BG, Thomley JE. 2017 Maternal androgens in avian brood parasites and their hosts: responses to parasitism and competition? Gen. Comp. Endocrinol. 240, 143-152. (doi:10.1016/j.ygcen.2016.10.004)

12. Ibáñez-Álamo JD, De Neve L, Roldán M, Rodríguez J, Trouvé C, Chastel O, Soler M. 2012 Corticosterone levels in host and parasite nestlings: is brood parasitism a hormonal stressor? Horm. Behav. 61, 590-597. (doi:10.1016/j.yhbeh.2012.02.008)

13. MacDougall-Shackleton SA, Bonier F, Romero LM, Moore IT. 2019 Glucocorticoids and "stress" are not synonymous. Integr. Org. Biol. 1. (doi:10.1093/iob/obz017)

14. Voellmy IK, Goncalves IB, Barrette M-F, Monfort SL, Manser MB. 2014 Mean fecal glucocorticoid metabolites are associated with vigilance, whereas immediate cortisol levels better reflect acute antipredator responses in meerkats. Horm. Behav. 66, 759-765. (doi:10.1016/j.yhbeh.2014.08.008)

15. Horton BM, Holberton RL. 2009 Corticosterone manipulations alter morph-specific nestling provisioning behavior in male white-throated sparrows, Zonotrichia albicollis. Horm. Behav. 56, 510-518. (doi:10.1016/j.yhbeh.2009.09.001) 
16. Angelier F, Clément-Chastel C, Welcker J, Gabrielsen GW, Chastel O. 2009 How does corticosterone affect parental behaviour and reproductive success? A study of prolactin in blacklegged kittiwakes. Funct. Ecol. 23, 784-793. (doi:10.1111/j.1365-2435.2009.01545.x)

17. Scharf HM, Stenstrom K, Dainson M, Benson TJ, Fernandez-Juricic E, Hauber ME. 2019 Mimicrydependent lateralization in the visual inspection of foreign eggs by American robins. Biol. Lett. 15, 20190351. (doi:10.1098/rsbl.2019.0351)

18. Hauber ME, Dainson M, Luro A, Louder AA, Hanley D. In press When are egg-rejection cues perceived? A test using thermochromic eggs in an avian brood parasite host. Anim. Cogn. (doi:10.1007/s10071-019-01306-w)

19. Rowe KMC, Weatherhead PJ. 2009 A third incubation tactic: delayed incubation by american robins (Turdus Migratorius). The Auk 126, 141-146. (doi:10.1525/auk.2009.07210)

20. Palomino JJ, Martín-vivaldi M, Soler M, Soler JJ. 1998 Females are responsible for ejection of cuckoo eggs in the rufous bush robin. Anim. Behav. 56, 131-136. (doi:10.1006/anbe.1998.0771)

21. Breuner CW, Jennings JH, Moore IT, Orchinik M. 2000 Pharmacological adrenalectomy with mitotane. Gen. Comp. Endocrinol. 120, 27-34. (doi:10.1006/gcen.2000.7537)

22. Deviche P, Giraudeau M, Desaivre S. 2017 Experimental manipulation of corticosterone does not influence the clearance rate of plasma testosterone in birds. Physiol. Biochem. Zool. 90, 693043693043. (doi:10.1086/693043)

23. Breuner CW, Orchinik M, Hahn TP, Meddle SL, Moore IT, Owen-Ashley NT, Sperry TS, Wingfield JC. 2003 Differential mechanisms for regulation of the stress response across latitudinal gradients. Am. J. Physiol. 285, R594-600. (doi:10.1152/ajpregu.00748.2002)

24. Lattin CR, Pechenenko AV, Carson RE. 2017 Experimentally reducing corticosterone mitigates rapid captivity effects on behavior, but not body composition, in a wild bird. Horm. Behav. 89, 121-129. (doi:10.1016/j.yhbeh.2016.12.016)

25. Abolins-Abols M, Hauber ME. Preprint Proximate predictors of variation in egg rejection behavior by hosts of avian brood parasites. bioRxiv, 788505. (doi:10.1101/788505)

26. Pyle P. 1997 Identification guide to North American birds. Part 1, Columbidae to Ploceidae. Bolinas, CA: Slate Creek Press.

27. Casto JM, Nolan V, Ketterson ED. 2001 Steroid hormones and immune function: experimental studies in wild and captive dark-eyed juncos (Junco hyemalis). Am. Nat. 157, 408-420. (doi:10.1086/319318)

28. Briskie JV, Sealy SG, Hobson KA. 1992 Behavioral defenses against avian brood parasitism in sympatric and allopatric host populations. Evolution 46, 334-340. (doi:10.1111/j.15585646.1992.tb02041.x)

29. Hanley D, Grim T, Igic B, Samaš P, Lopez AV, Shawkey MD, Hauber ME. 2017 Egg discrimination along a gradient of natural variation in eggshell coloration. Proc. R. Soc. B Biol. Sci. 284, 201625920. (doi:10.1098/rspb.2016.2592) 
30. Luro AB, Hauber ME. 2017 A test of the nest sanitation hypothesis for the evolution of foreign egg rejection in an avian brood parasite rejecter host species. Sci. Nat. 104, 14. (doi:10.1007/s00114-0171446-8)

31. Grim T, Samaš P, Moskát C, Kleven O, Honza M, Moksnes A, Røskaft E, Stokke BG. 2011 Constraints on host choice: why do parasitic birds rarely exploit some common potential hosts? J. Anim. Ecol. 80, 508-518. (doi:10.1111/j.1365-2656.2010.01798.x)

32. Croston R, Hauber ME. 2014 High repeatability of egg rejection in response to experimental brood parasitism in the American robin (Turdus migratorius). Behaviour 151, 703-718. (doi:10.1163/1568539X-00003164)

33. Chen Y-Z, Qiu J. 1999 Pleiotropic signaling pathways in rapid, nongenomic action of glucocorticoid. Mol. Cell Biol. Res. Commun. 2, 145-149. (doi:10.1006/mcbr.1999.0163)

34. Sapolsky RM, Romero LM, Munck AU. 2000 How do glucocorticoids influence stress responses? Integrating permissive, suppressive, stimulatory, and preparative actions. Endocr. Rev. 21, 55-89. (doi:10.1210/edrv.21.1.0389)

35. Garamszegi LZ, Rosivall B, Rettenbacher S, Markó G, Zsebők S, Szöllösi E, Eens M, Potti J, Török J. 2012 Corticosterone, avoidance of novelty, risk-taking and aggression in a wild bird: no evidence for pleiotropic effects. Ethology 118, 621-635. (doi:10.1111/j.1439-0310.2012.02049.x)

36. Breuner CW, Hahn TP. 2003 Integrating stress physiology, environmental change, and behavior in free-living sparrows. Horm. Behav. 43, 115-123. (doi:10.1016/S0018-506X(02)00020-X)

37. Landys MM, Goymann W, Schwabl I, Trapschuh M, Slagsvold T. 2010 Impact of season and social challenge on testosterone and corticosterone levels in a year-round territorial bird. Horm. Behav. 58, 317-325. (doi:10.1016/j.yhbeh.2010.02.013)

38. Spée M, Marchal L, Lazin D, Le Maho Y, Chastel O, Beaulieu M, Raclot T. 2011 Exogenous corticosterone and nest abandonment: A study in a long-lived bird, the Adélie penguin. Horm. Behav. 60, 362-370. (doi:10.1016/j.yhbeh.2011.07.003)

39. Crossin GT, Trathan PN, Phillips RA, Gorman KB, Dawson A, Sakamoto KQ, Williams TD. 2012 Corticosterone predicts foraging behavior and parental care in macaroni penguins. Am. Nat. 180, E31-E41. (doi:10.1086/666001)

40. Vitousek MN, Taff CC, Ardia DR, Stedman JM, Zimmer C, Salzman TC, Winkler DW. 2018 The lingering impact of stress: brief acute glucocorticoid exposure has sustained, dose-dependent effects on reproduction. Proc. R. Soc. B Biol. Sci. 285, 20180722. (doi:10.1098/rspb.2018.0722)

41. Bowers EK, Bowden RM, Thompson CF, Sakaluk SK. 2016 Elevated corticosterone during egg production elicits increased maternal investment and promotes nestling growth in a wild songbird. Horm. Behav. 83, 6-13. (doi:10.1016/j.yhbeh.2016.05.010) 


\section{Figures}

311 Figure 1. Treatment validation in wild-caught, captive American robin females. Mitotane-treated birds

312 (orange round dots) showed lower increase in corticosterone levels compared to the sham-treated birds

313 (grey triangles). Samples taken from the same individual are connected with lines.

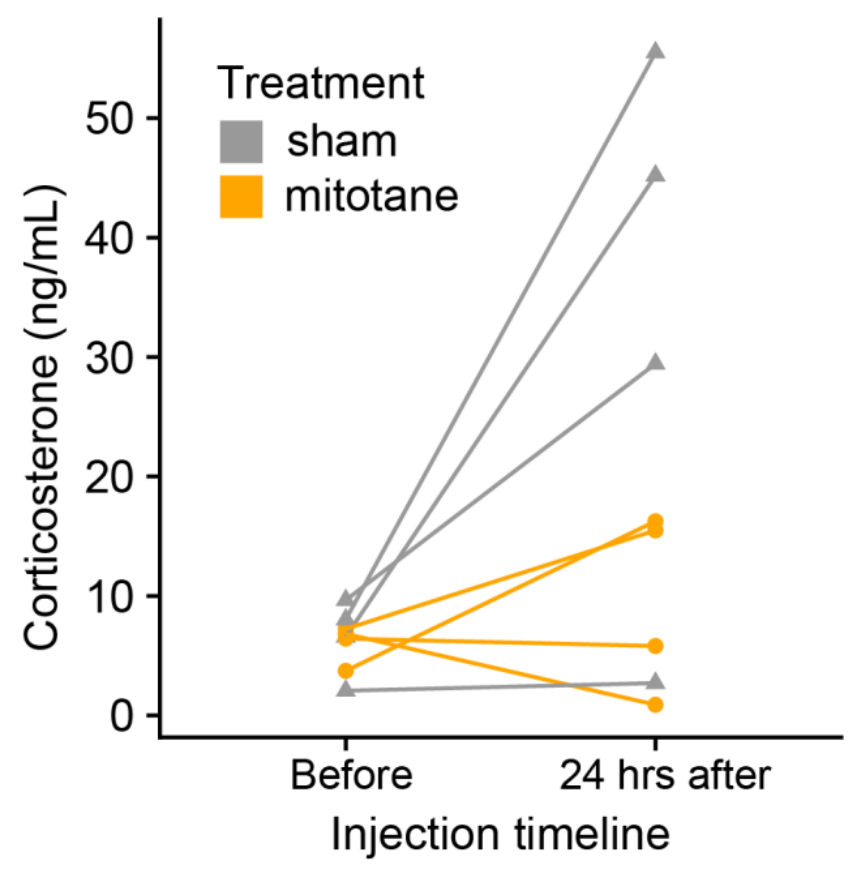


317 Figure 2: The effect of mitotane on non-mimetic model egg acceptance. Mitotane-treated birds (orange)

318 were significantly more likely to accept model eggs compared to the sham-treated birds (grey). The dotted

319 line indicates a 50\% acceptance probability. The top-left insert shows the non-mimetic deep-blue model

320 egg (right) next to natural American robin eggs (left). Numbers at the top reflect the total number of birds

321 in each treatment.

322

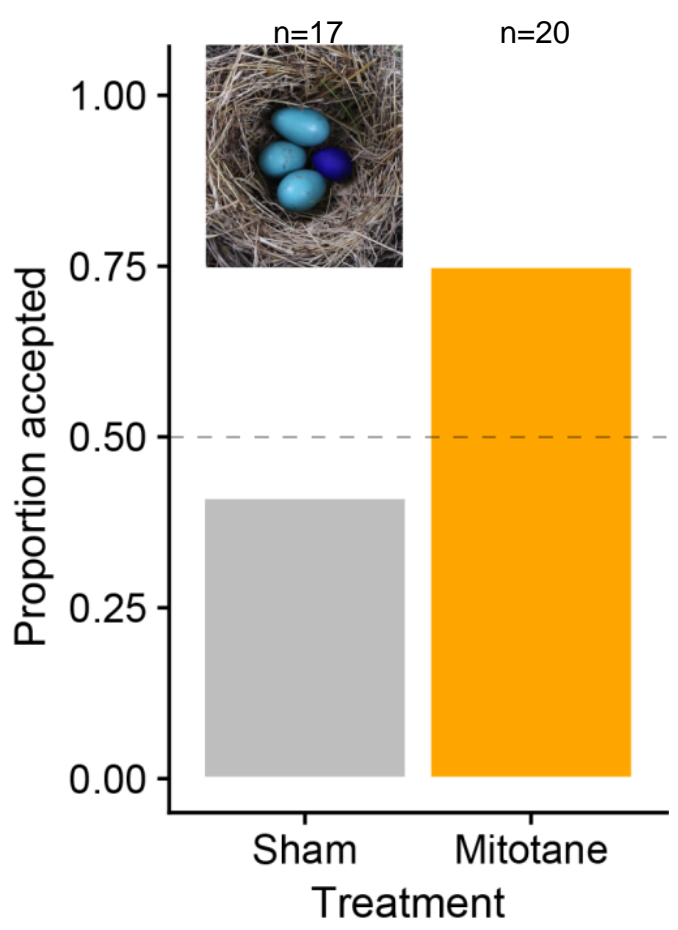

